\title{
Routing Analysis and Energy Efficiency in Wireless Sensor Networks*
}

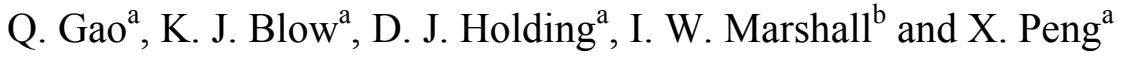 \\ ${ }^{a}$ Electronic Engineering Department, Aston University, Birmingham B4 7ET, United Kingdom \\ \{qianggao, k.j.blow, d.j.holding, x-h.peng\}@aston.ac.uk \\ ${ }^{b}$ BTexacT Technologies, Adastral Park, Martlesham Heath, Ipswich, Suffolk IP5 3RE, United Kingdom \\ ian.w.marshall@bt.com
}

\begin{abstract}
We combine a simple energy model of radio transceivers with analysis of ad-hoc routing to optimize system performance. A simple example of a linear wireless ad hoc sensor network is used to deduce the relationship between traffic load and optimal radio range of topology management schemes. We find that half of the power can be saved if the radio range is adjusted appropriately compared with the best case where equal radio ranges are used.
\end{abstract}

Keywords-Wireless sensor networks, energy efficiency, topology management, radio range adjustment.

\section{INTRODUCTION}

Recent advances in micro-electro-mechanical systems (MEMS) technology, wireless communications and digital electronics have enabled the development of low-cost, lowpower, multifunctional smart sensor nodes. Smart sensor nodes are autonomous devices equipped with heavily integrated sensing, processing, and communication capabilities. When these nodes are networked together in an ad-hoc fashion, they form a sensor network. One of the key challenges in unlocking the potential of data gathering sensor networks is conserving energy so as to maximize their post-deployment active lifetime [1][5].

Topology management schemes such as GAF [8][10], SPAN [4], ASCENT [3], CEC [11] and AFECA [9] take advantage of high-density deployment of sensor networks to save energy by electing a few nodes to perform routing while allowing the remaining nodes to enter a sleep state. In SPAN, a limited set of nodes forms a multi-hop forwarding backbone, which tries to preserve the original capacity of the underlying ad-hoc network. Other nodes transition to sleep states more frequently, as they no longer carry the burden of forwarding data of other nodes. To balance out energy consumption, the backbone functionality is rotated between nodes and therefore there is a strong interaction with the routing layer. In ASCENT, the decision for being active is the courtesy of the node. Passive nodes keep listening all the time and assess their course of actions; stay passive or be- come active. Cluster-based Energy Conservation (CEC) and the Adaptive Fidelity Energy-Conserving Algorithm (AFECA) are two other proposed energy conserving topology management algorithms. CEC creates clusters and selects cluster-heads based on the highest advertised remaining energy. AFECA allows each node to sleep for randomized periods based on the number of (overheard) neighbors it has.

In this paper we deduce the relationship between optimal radio range and traffic and use this to define a non-uniform grid for the GAF protocol, and show that this has better performance than the uniform grid.

\section{GAF Algroithm AND RADIO POWER MODEL}

The GAF algorithm is based on a division of the sensor network into a number of virtual grids of size $\mathrm{R}$ by $\mathrm{R}$, see Fig. 1. The value of $\mathrm{R}$ is chosen such that all nodes in a grid are equivalent from a routing perspective. This means that any two nodes in adjacent grids should be able to communicate with each other. By investigating the worst-case node locations depicted in Fig. 1, we can calculate that $\mathrm{R}$ should satisfy

$$
R \leq \frac{r}{\sqrt{5}}
$$

where $\mathrm{r}$ is radio range. For the one dimension case, $\mathrm{R}$ should satisfy

$$
R \leq r / 2
$$

If we assume that the energy demanded for transmission is independent of the distance then longer system lifetime can be achieved by increasing the number of nodes in a grid section, thereby increasing the number of sleeping nodes, when we increase the radio range. In practice, this is not the case and we now introduce an energy use model that can be combined with analysis of the routing protocol to optimize the system performance.

\footnotetext{
* This research was supported by BT Advanced Communications Research Group, BTExacT, U.K under contract ML847381 "Active Networks and the Physical Layer".
} 


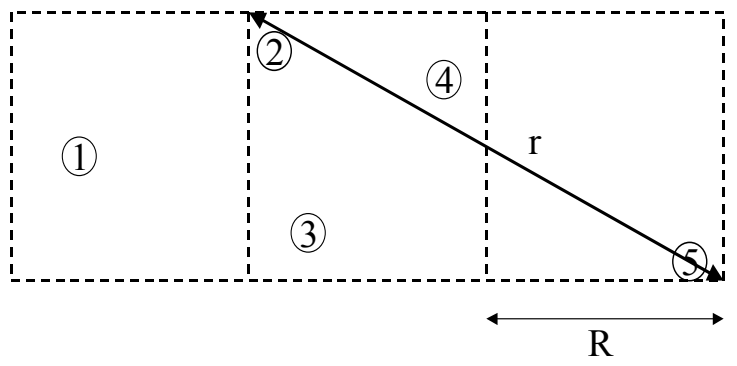

Fig. 1. GAF virtual grid structure.

Airborne radio transmissions are attenuated by path loss at a rate which scales as a power law with distance [7]. The energies consumed per second in transmission and reception are respectively:

$E_{t}=\left(e_{t}+e_{d} r^{n}\right) B, E_{r}=e_{r} B$

where $e_{t}, e_{d}$ and $e_{r}$ are properties of the transceiver used by the nodes, and $r$ is the transmission range used. The parameter $n$ is the power index for the channel path loss of the antenna, a factor that depends on the RF environment, and is generally between 2 and $4 . B$ is the bit rate of the radio. Typical numbers for current radios are

$e_{t}=e_{r}=50 \times 10^{-9}$ Joules / bit ,

$e_{d}=100 \times 10^{-12}$ Joules $/$ bit $/ m^{2}(n=2)$,

$B=1$ Mbit $/$ sec $[6]$.

Since the path loss of radio transmission scales with distance in a greater-than-linear fashion the total transmission energy can therefore be reduced significantly using a multihop approach (dividing a long transmission path into several shorter ones). Now the problem is how can we reach the optimal range for energy efficient routing that uses the smallest amount of energy for data transmission while simultaneously allowing many nodes to be put into the sleep state.

\section{RELATIONSHIP BETWEEN RANGE AND TRAFFIC}

Consider first a transport network where there is data transmission over distance $d$ from a source to a destination. The traffic in Erlang is $A$. If the transmission route is divided into a grid of $k$ sections and only one node wakes up in each section as relay node (note the standard GAF protocol is now assumed for this analysis) then the total energy consumption per second by $k$ hops is

$$
P=k\left[e_{r} B A+e_{t} B A+e_{d}\left(2 \frac{d}{k}\right)^{n} B A+c e_{r}(1-2 A) B\right]
$$

The last term in (4) represents the energy consumption during the idle state and the energy consumption ratio idle: receive $=c: 1$. The energy efficient optimum size of the virtual grid section can now be derived and is given by

$$
R_{o p t}=r_{o p t} / 2=\sqrt[n]{\frac{\left(e_{r}+e_{t}\right) A+c e_{r}(1-2 A)}{2^{n} A(n-1) e_{d}}}
$$

The minimum energy consumption characteristic range is no longer a constant as in [2] but changes with the amount of traffic. Fig. 2 shows the relationship between the traffic $A$ and the optimal range $r_{\text {opt }}$. The optimal range decreases as the loaded traffic increases. At the extreme point $\mathrm{A}=0.5$, where the transmitter spends $50 \%$ of the time transmitting and $50 \%$ receiving (we assume the node can only do one or the other), there is no idle time and so the optimal range converges to the characteristic range $d_{c h a r}$ in [2]. Under conditions of light traffic the optimal range increases sharply as the loaded traffic decreases. When the data transferred in the sensor network is low, the idle state dominates the energy consumption and hence the radio range can be relatively large.

\section{TRANSMISSION RANGE AJUSTMENT}

In many applications of wireless sensor networks, data is gathered by multiple sensors at different locations and transmitted to a single sink node (such as a base station) where data can be stored and analyzed. Consider a linear network where nodes are deployed uniformly between the edge and the sink. The network is divided into a grid, each section of which contains a single routing node. The routing node in each section receives data directly from the non-routing nodes in the adjacent upstream section, as well as relayed traffic from the upstream routing node, and in turn relays it to the routing node in the adjacent downstream section. The base station replaces the routing node in the first section and the final section contains no routing node. If each node produces $a$ Erlang of sensor data then the traffic needed to be forwarded at a point $x$ meters from the base station is

$A(x)=(d-x) n_{d} a$

where $d$ is the total length of the one-dimensional network and $n_{d}$ is the node density.

To save energy by radio range adjustment we divide the network into sections of different size as shown in Fig. 3 according to the range-traffic relationship (5). The sizes of 
the grid sections and radio ranges can be calculated iteratively according to the following heuristic algorithm where the optimum grid section size of the traffic dependent transport network (6) is matched to the local traffic in the sensor network.

$$
\begin{aligned}
& R_{1}=R_{o p t}\left(x=R_{1}\right) \\
& R_{2}=R_{o p t}\left(x=R_{2}+R_{1}\right) \\
& \cdots \\
& R_{i}=R_{o p t}\left(x=\sum_{j=1}^{i} R_{j}\right)
\end{aligned}
$$

and

$$
r_{1}=R_{1}, r_{2}=R_{2}+R_{1}, \cdots, r_{i}=R_{i}+R_{i-1}
$$

In the case where the length of the linear network is $d=600 \mathrm{~m}$, node density $n_{d}=1 / 6$ per meter and every node produces data of $\mathrm{a}=0.003$ Erlang, the heuristic nonuniform grid section sizes are calculated as Table 1 .

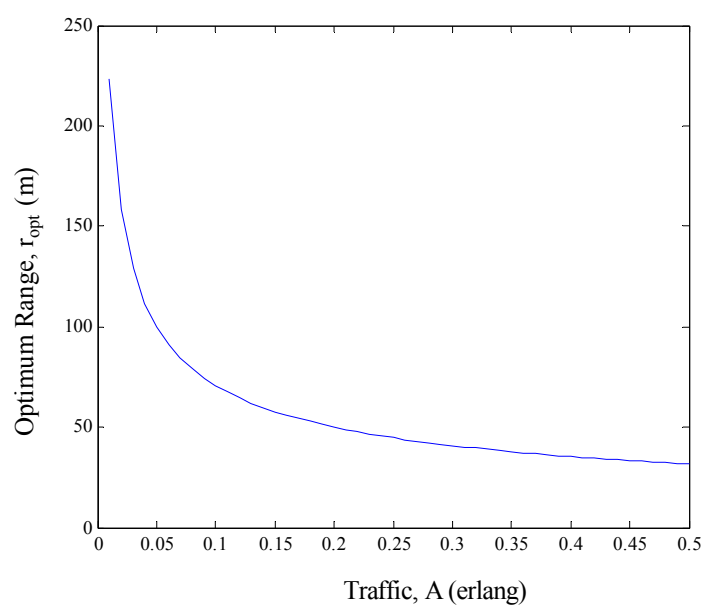

Fig. 2. Optimal range-traffic relationship.

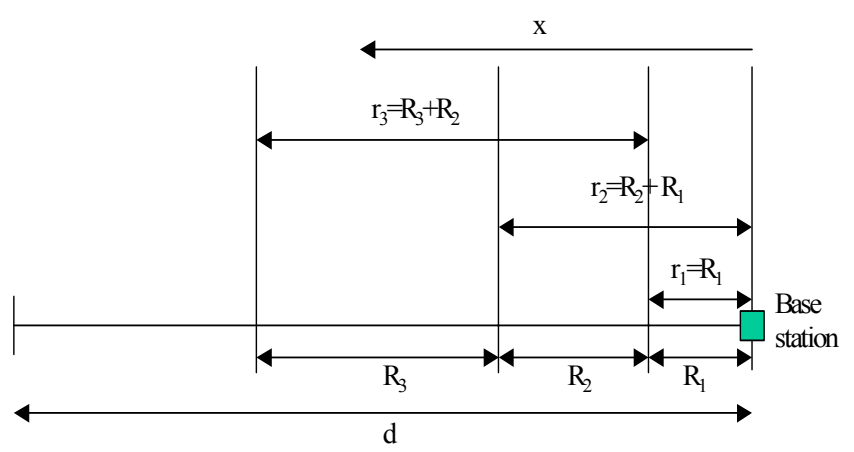

Fig. 3. A linear network covered by a virtual grid with sections of different size.
TABLE I

Grid Sizes According to Heuristic Algorithm (7)

\begin{tabular}{|l|l|l|l|l|l|l|l|}
\hline $\mathrm{i}$ & 1 & 2 & 3 & 4 & 5 & 6 & 7 \\
\hline $\mathrm{R}_{\mathrm{i}}(\mathrm{m})$ & 20.8 & 21.2 & 21.6 & 22.0 & 22.5 & 23.1 & 23.7 \\
\hline $\mathrm{i}$ & 8 & 9 & 10 & 11 & 12 & 13 & 14 \\
\hline $\mathrm{R}_{\mathrm{i}}(\mathrm{m})$ & 24.4 & 25.1 & 26.0 & 27.0 & 28.2 & 29.6 & 31.4 \\
\hline $\mathrm{i}$ & 15 & 16 & 17 & 18 & 19 & & \\
\hline $\mathrm{R}_{\mathrm{i}}(\mathrm{m})$ & 33.7 & 37.0 & 42.1 & 53.7 & 86.7 & & \\
\hline
\end{tabular}

The energy consumptions of reception, transmission and listening in the ith grid section are respectively

$$
\begin{aligned}
& E_{r}(i)= e_{r}\left(d-R_{1}-\ldots-R_{i}\right) n_{d} a B, \\
& E_{t}(i)=\left\{\begin{array}{l}
\left(e_{t}+e_{d} R_{1}^{n}\right) d n_{d} a B, i=1 \\
{\left[e_{t}+e_{d}\left(R_{i}+R_{i-1}\right)^{n}\right]\left(d-R_{1}-\ldots-R_{i-1}\right) n_{d} a B, i>1}
\end{array}\right.
\end{aligned}
$$

$E_{\text {idle }}(i)=c e_{r}\left[1-2\left(d-R_{1}-\ldots-R_{i}\right) n_{d} a\right] B$

The total energy consumption of the whole network is now obtained by summing these contributions over the entire grid

$E=\sum_{i=1}^{k} E_{r}(i)+E_{t}(i)+E_{\text {idle }}(i)$

For the case considered, if we assume that the energy consumption in the idle state is approximately equal to that in the receiving state (i.e. $c=1$ ), then the total network energy consumption is $E=9.5102 \times 10^{-4}$ Joules $/ \mathrm{sec}$.

The energy consumption for the non-uniform grid is shown as the isolated point in Fig. 4. Note that the number of grid sections in the network is not a free parameter in the non-uniform grid unlike the standard GAF protocol. The total energy consumption for a uniform grid is also plotted versus the number of sections in Fig. 4. We see that lower energy consumption is achieved by the non-uniform virtual routing grid constructed according to the range-traffic relationship as compared to the optimum value for the uniform grid. The lowest energy consumption for the equal radio range case is $2.1 \times 10^{-3}$ Joules / sec . So about $50 \%$ energy is saved if we use adaptive grid division. If we choose $c=2$, that is receiving consumes twice as much energy as listening, the total energy consumption of the whole sensor network for unequal grid division case is $1.4 \times 10^{-3}$ Joules / sec and the lowest energy consumption for equal grid division case is $2.7 \times 10^{-3}$ Joules / sec . Thus there is no significant change of our result if we change the energy consumption ratio of idle:receive. 


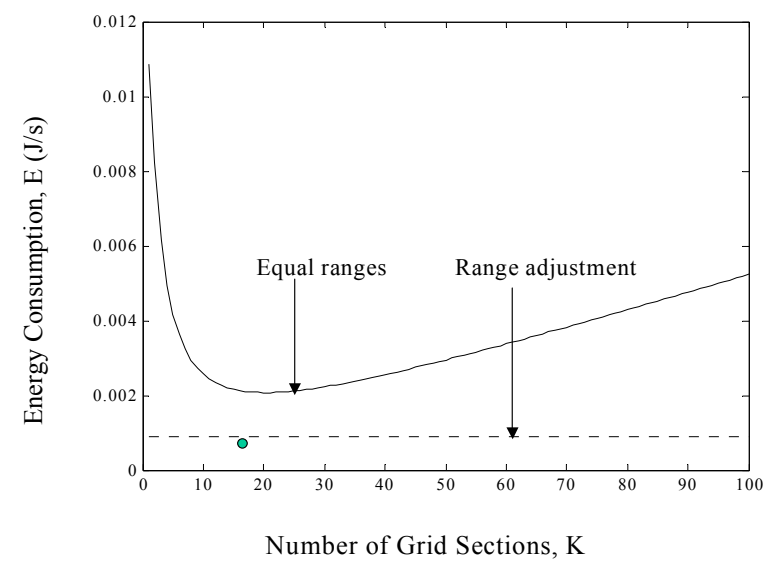

Fig. 4. Comparison of the total energy consumption for adaptive and uniform grids as a function of the number of grid sections for the uniform grid case.

\section{CONCLUSIONS}

In this paper we proposed a novel method of analyzing the energy efficiency performance of ad-hoc sensor networks. This has been used to show that radio range adjustment can save energy when combined with a topology management scheme. Taking a simple one dimension network and the GAF protocol as an example we deduced the relationship between optimal radio range and traffic and find that half of the power can be saved if the radio range is adjusted appropriately in a linear network. The results show that adjusting the radio range to adapt for network properties and traffic is an important consideration for the optimization of ad-hoc sensor networks. Our results should be expanded to two dimension networks and hierarchical (cluster) networks. The relationship between optimal radio range and traffic can be modified to apply to other topology management protocols. When we adjust radio ranges to save energy the unidirec- tional links are introduced, which provide a challenge to routing algorithms.

\section{REFERENCES}

[1] I. F. Akyildiz, W. Su, Y. Sankarasubramaniam, and E. Cyirci, "Wireless sensor networks: a survey," Computer Networks, vol. 38, pp. 393 422, March 2002.

[2] M. Bhardwaj, G. Garnett, and A.P. Chandrakasan, "Upper bounds on the lifetime of sensor networks," in Proc. ICC 2001, vol. 3, pp. 785 790, Helsinki, Finland, June 2001.

[3] A. Cerpa, and D. Estrin, "ASCENT: adaptive self-configuring sensor networks topologies," in Proc. INFOCOM 2002, vol. 3, pp. 1278-1287, New York, US, June 2002.

[4] B. J. Chen, K. Jamieson, H. Balakrishnan, and R. Morris, "Span: an energy-efficient coordination algorithm for topology maintenance in Ad Hoc wireless networks," Wireless Networks, vol. 8, pp. 481-494, September 2002.

[5] Q. Gao, K.J. Blow, D.J. Holding, and I. Marshall, "Determining design parameters for ad hoc wireless sensor networks," in Proc. IEEE ETFA'2003, vol. 1, pp. 545-550, Lisbon, Portugal, September 2003.

[6] W. R. Heinzelman, A. Chandrakasan, and H. Balakrishnan, "Energyefficient communication protocol for wireless microsensor networks," in Proc. HICSS '00, pp. 4-7, Hawaii, US, January 2000.

[7] T. Rappaport, Wireless Communications: Principles \& Practice, Prentice-Hall, Inc., New Jersey, 1996.

[8] C. Schurgers, V. Tsiatsis, S. Ganeriwal, and M.B. Srivastava, "Optimizing sensor networks in the energy-latency-density design space," IEEE Transactions on Mobile Computing, vol.1, pp. 70-80, JanuaryMarch 2002.

[9] Y. Xu, "Adaptive energy-conserving routing for multihop ad hoc networks, " USC/ISI Research Report TR-2000-527, October 2000.

[10] Y. Xu, J. Heidemann, and D. Estrin, "Geography-informed energy conservation for Ad Hoc routing," in Proc. ACM/IEEE Int. Conf. on Mobile Computing and Networking, pp. 70-84, Rome, Italy, July 2001.

[11] Y. Xu, J. Heidemann, and D. Estrin, "Energy conservation by adaptive clustering for ad-hoc networks," in Poster Session of MobiHoc 2002, pp. 255-263, Lausanne, Switzerland, June 2002. 